\title{
Exploration on the Influence of Physical Exercise on Physical and Mental Health and its Mechanism
}

\author{
Pengju Guo \\ Department of Physical Education, Xi'an Peihua University, Xi'an Shanxi, 710125, China
}

Keywords: Physical exercise, Influence on physical and mental health, Physical exercise mechanism.

\begin{abstract}
In recent years, the country has attached importance to college students' physical fitness and mental health, so the management personnel concerned need to perfect the physical exercise mechanism in colleges. Besides, students' learning interest and physical exercise habit should serve as important items in curriculum. To some extent, promoting students' harmonious physical and mental development and raising college students' overall health level can practically train students better and help them to develop into a better innovative habit. Furthermore, physical education can help students to cultivate their enterprising spirit. Therefore, based on the analysis on the influence of physical exercise in colleges on physical health, this paper came up with some measures for mechanism perfection so as to provide PE teachers concerned with references.
\end{abstract}

\section{Introduction}

When college students participate in physical exercise, management personnel concerned should pay attention to the influence on students' physical and mental health, take effective measures to formulate a complete system, require students to form a good living habit and physical exercise habit, and guarantee to better improve the mode of physical exercise so as to help students to obtain better development and lay a favorable foundation for the development of students' physical and mental health.

\section{Influence of physical exercise on college students' physical and mental health}

During the physical teaching process in colleges, students' physical and mental health is extremely important, so it is required to fully understand the influence of physical exercise on college students' physical and mental health for the purpose of improving the efficiency of physical exercise. It is specifically shown below:

\section{To influence college students' level of intelligence}

For college students, intelligence directly determines their learning efficiency and later working quality. The fierce competition in national market has made a higher demand for college students' comprehensive quality, so it is required to improve college students' comprehensive quality and make effective reforms in teaching. Especially, college students are in a high-tension learning state, which may give rise to college students' mental and physical exhaustion if no adjustment is made; furthermore, this may also has adverse effects on their development and even will cause them to fall ill. Therefore, sports activities carried out in colleges can release students' tension and, meanwhile, train college students' intelligence so that they can relieve their pressure in a better way. As shown in the researches made by overseas scientists, physical exercise can effectively improve human's cardiopulmonary function; especially, human's cerebral function can be increased by $15 \%$. According to the experiment on a hundred volunteers, fifty of them were asked to take physical exercise for seven months and the other fifty ones were in the state without physical exercise. The experiment found that the 7-month physical exercise could better improve the physical health and also enhance the intelligence while those without physical exercise had their brain mechanism affected and 
suffered physical illness. Therefore, after physical exercise provided in colleges can effectively stimulate students' learning interest, cultivate their taste and train their intelligence to some extent.

\section{To influence college students' emotion}

As shown in the researchers made by people concerned, one who usually takes exercise can secrete the peptides substance which can influence his psychological state through physical exercise and this substance can excite him and make him happy; then, one can effectively improve both his learning and working quality. During physical exercise, colleges can better adjust college students' central nervous system, make students feel excited and help them to learn how to control their emotion. Meanwhile, after physical exercises and activities are carried out in colleges, students' mental depression can be relieved effectively; besides, through physical exercise, students can accelerate their blood circulation, quicken breath and relieve their tension. Therefore, carrying out healthy sports activities in colleges can better increase students' pleasure, keep up their spirits, effectively cultivate their tastes, relieve their mental unrest and loneliness to some extent, eliminate their inferiority complex and lay a favorable foundation for their development ${ }^{[1]}$.

\section{To influence college students' self-awareness}

Physical exercise carried out in colleges can affect students' self-awareness. College students are in a special age period, which is exactly the puberty for their psychological development, so they have a strong need for emotional exchange and cannot deal with the interpersonal relationship well. Besides, it is required to cultivate college students' health personality, which is namely that one can keep the mutual harmony between their psychology and behavior, be positive and can effectively govern their behavior and psychology during the process of psychological development. Physical exercise requires students to better comply with the Olympic spirit, so that they can restrain their behaviors in all sorts of contests and regulate their psychological states. Thus, it can be seen that colleges can better cultivate college students' personality after carrying out physical exercise so that students will have more confidence, have strong self-esteem and effectively establish their self-awareness so as to lay a favorable foundation for their development ${ }^{[2]}$.

\section{To influence college students' social adaptability}

Due to the influence of social environment, it is difficult to cope with the interpersonal relationship and people fail to make emotional exchanges well, causing complicated interpersonal relationship and other issues, which will have adverse effects on college students' development; then, college students easily become unsociable and depressed and the learning atmosphere is depressing so that students' comprehensive quality cannot be improved. At this time, college students need some activities to relieve their stress. Physical exercises and activities carried out in colleges can enhance students' social adaptability to some extent, because college students need to cooperate with their team members when participating in physical exercise; during cooperation, a favorable interpersonal relationship can be formed among them and thus they can cope with all sorts of interpersonal problems and attain the goal of cultivating college students' social adaptability. For instance, when a sports competition activity is held in a college, students will be evaluated for their sports techniques through group activities. In this way, not only students' team collaboration skills can be cultivated, but also their desire for physical exercise can be stimulated, so they can have the fighting spirit, correctly cope with interpersonal relationship and guarantee to better coordinate their own behaviors. When a sports activity is carried out in colleges, students can exchange their information with only a gesture or an eye contact; then, they can have the sense of group honor and their development can also be promoted accordingly. Thus, it can be seen that physical exercise in colleges can better cultivate college students' psychological quality and help them to develop in a healthy way. The Ministry of Education's reform in physical education and higher requirements for physical education not only can enhance students' physical skills but also cultivate students' psychological quality; besides, students can face the trend of social completion with a healthy and positive attitude. On the basis of strengthening students' physical quality, these can also facilitate students' psychological development, 
have more positive influences on students and cultivate a lot of talents with higher comprehensive quality ${ }^{[3]}$.

\section{Influence of physical exercise in colleges on physical heath mechanism}

At present, only a few people have studied the influence of physical exercise on the physical health mechanism and the conclusions made by them through researches are inconsistent. It can be found through the analysis on the research conclusions that human's control over diet in combination with sports and taking lipid-lowering drugs can well decelerate the growth of fat, greatly consume fat and thus reach the effect of medical treatment; in addition, the reduction in fact can effectively prevent diseases arising from obesity. This conclusion is one of the mechanisms that physical exercise influences psychological health. Secondly, after taking physical exercise, diabetic patients can better control their blood glucose and effectively prevent complication. However, there are many divergences in the mechanism of action of physical exercise for diabetic patients and no consistent conclusion is available now, so careful researches by experts concerned are still required. Thirdly, some experts hold the opinion that sports can adjust the endocrine, because human's endocrine can be effectively improved after exercise to promote the skeleton metabolism, which thus can help human to indirectly adjust their skeletons and have very favorable influences on their physical health. Then, the exercise with an ordinary intensity cannot obviously stimulate the improvement in the internal secretion of human bodies and only enlarging the intensity of exercise can reach the effect of adjusting skeletons. Finally, exercise can effectively promote human's anti-depression rate. After taking an exercise, the strong neurotrophic factors generated after exercise can better adjust human's emotion; a large quantity of exercises can effectively enhance one's aerobic working ability and reach the purpose of mental health. However, in such researches, experts haven't confirmed whether sports can senility and vast researches are required ${ }^{[4]}$.

Some overseas experts found through researches on endurance exercise that osteoprotegerin can be generated after endurance exercise, because the osteocyte will secrete the component which will influence the skeletal development after endurance exercise and this component is the important mechanism to maintain skeletal balance. Meanwhile, in the process of studying endurance exercise, it was found that, after marathon runners finished their exercise, their skeleton can reabsorb nutrients to attain the goal of reconstruction with the influence of stimulating metabolism in the short run. It should be noted that this is the analytical result of a short-time influence, the long-term influence needs to be further studied ${ }^{[5]}$.

\section{Measures for perfection of college physical exercise mechanism}

Colleges can help students' psychological development and help them to have team spirit by carrying out physical exercise, through which, they can not only obtain healthy development but also enhance their interpersonal skills and improve their social competitiveness. Therefore, college PE teachers should attach importance to physical exercises and activities and formulate a perfect physical exercise mechanism to facilitate the development of students' comprehensive quality. The specific measures for mechanism perfection are shown below:

\section{To improve the lifestyle}

During the process of carrying out physical exercise, colleges should attach importance to students' lifestyle. As shown in related researches, human's lifestyle influences one's own health. A healthy lifestyle can better improve one's physical health. The extension of human longevity is directly related to one's lifestyle, so a good living habit can better prolong one's longevity. On the contrary, a bad living habit will cause all sorts of diseases and affect one's physical health or even result in death. Scientists concerned also found in their research processes that human beings with different lifestyles are also different in their level of physical health. Smokers and alcoholics suffer severe health problems. There are many smokers among China's college students every year; if no timely treatment 
is made for this, it will cause adverse effects on college students' physical health. Especially, after alcoholic intemperance, college students cannot to do physical exercises with their healthy bodies, causing increasingly poor body functions. Therefore, colleges should formulate a strict system for the management over students and timely punish any student, who is found with smoking or alcoholic intemperance problem, so as to avoid unfavorable influences on college students' physical health.

\section{To improve the habit of exercise}

As shown in experts' research processes, occasional exercises and increasingly fierce exercises will result in the problems of strong beating of the heart, rapidly rising blood pressure and growing artery resistance, all of which may induce various diseases. Especially, when one takes exercise in the starving condition, all his sense organs will suffer pessimal stimulation, which will not only damage his locomotive organ but also result in the functional disorder of human body; this will have adverse effects on him and even shorten the life of his organ. Therefore, college management personnel should pay attention to improving students' habit of exercise and make phased improvements on their habit of exercise. In addition, PE teachers should require college students to get engaged in collective activities, guarantee to improve their physical function through correct labor, and carry out physical exercises every day so that students can take physical exercise under the correct exercise guidance and won't damage their physical function due to occasional exercise. Besides, they can adjust their physical health and eliminate their overfatigue through scientific and reasonable exercise, which will also have favorable influences on their development.

\section{To formulate a perfect exercise scheme}

In order to better improve college students' physical and mental health through physical exercise, colleges need to formulate a perfect exercise scheme to help students to participate in physical exercise in a better way. In the research process, an American expert put forward an exercise prescription, which includes five elements which are namely setting of sports event, proper control over exercise intensity, favorable setting of exercise time, control over exercise frequency and regulation of exercise progress. The exercise prescription can effectively improve human's physical training condition, so colleges may introduce such a prescription as the foundation for the formulation of an exercise scheme. While formulating the exercise scheme, college management personnel should attach importance to the following work: firstly, comprehensively evaluate students' individual physical constitutions and guarantee to make exercise events suitable for students' physical development. Secondly, after selection of exercise events, reasonably control the exercise intensity and guarantee to better control the exercise frequency. Thirdly, PE teachers may make exercise schemes based on different constitutions of different students, guarantee to be based on strengthening students' physique, enhance the influence of physical exercise on physical health and reduce the incidence of diseases.

\section{Conclusion}

Physical exercise in colleges directly affects students' physical and psychological development, so PE teachers should introduce advance exercise technologies based on the analysis on relevant researches and formulate perfect exercise schemes for students so that students can enhance the efficiency of their physical health development through physical exercise. In addition, colleges should pay attention to PE teachers' professional qualities and establish a correct concept of physical exercise so as to lay a foundation for the development of students' physical and mental health.

\section{References}

[1] Gu Haixiang. Brief discussion of the influence of vocational schoolgirls' participation in physical exercise on their physical and mental health, Science \& Technology Information, 2012(22):300. 
[2] Xiong Zhengying and Wang Yimin. One of difficulties in theoretical teaching of high school PE teachers - physical exercise as well as physical and mental health, Contemporary Teacher Education, 2012(1):68-72.

[3] Yang Yongqiang. Brief discussion on the relationship between physical exercise and physical and mental health, Space Time of Sports, 2012 (3): 26.

[4] Long Hewen. Analysis on the influence of the urban old people's participation in physical exercise in the concentrated areas of nationalities on their physical and mental health - taking the Honghe Hani and Yi Autonomous Prefecture of Yunnan for example, Bulletin of Sport Science \& Technology, 2012,20 (7): 69-70, 81.

[5] Lu Lijuan. Analysis on the current situation of physical exercise and physical and mental health of higher adult education students and countermeasures, Modern Enterprise Education, 2012 (18):106-107. 